Reprod. Nutr. Dévelop., 1986, 26 (3), 841-847.

\title{
Effets de l'apport alimentaire en calcium sur la fonction thyroïdienne chez le rat
}

\author{
Nicole ETLING, Françoise FOUQUE, Michèle GARABEDIAN
}

INSERM U.30 \& C.N.R.S., UA. 583 ,

Hôpital des Enfants-Malades,

149, rue de Sèvres, 75743 Paris Cédex 15.

Summary. Effects of calcium supply in food on thyroid function in the rat.

To determine if calcium had a goitrogenic effect on the thyroid function in rats, weanling rats were fed, for three weeks, a diet containing either (a) $0.5 \mu \mathrm{g}$ or $0.04 \mu \mathrm{g}$ iodine per gram of diet, or (b) an adequate $(0.47 \%)$ or an excessive $(2 \%)$ amount of calcium.

With an adequate iodine diet, the calcium load did not induce an increase in the weight of the thyroid or a decrease in serum thyroid hormone concentration. However, the rats given a calcium load had a lighter body weight and a lower iodine content in the thyroid tissue; they also had a higher thyroxine $\left(T_{4}\right)$ content in the liver and kidney tissues than the rats receiving an adequate calcium diet.

With a low iodine diet, the calcium load brought out a decrease in growth and a lower serum triiodothyronine $\left(T_{3}\right)$ concentration and liver and kidney $T_{3}$ contents. These changes suggest that the calcium load might have acted on the thyroid function through an inhibition of $\mathrm{T}_{4}-\mathrm{T}_{3}$ conversion in the serum as well as in liver and kidney tissues.

\section{Introduction.}

Les revues générales sur les substances goitrogènes (Langer et Greer, 1977) citent encore le calcium et ajoutent que son mécanisme d'action n'est pas élucidé. Boussingault (1831) recherchant la cause des goitres dans les populations vivant dans la Cordillère des Andes avait suggéré que la forte teneur en calcium de l'eau de boisson pouvait en être la cause. De même, Sharpless et al. (1943) pensaient que le calcium, en présence de vitamine $D$, pouvait agir comme un goitrogène. Enfin Taylor (1954), étudiant l'histologie du tissu thyroïdien de rats nourris avec un régime riche en calcium et pauvre en iode, montrait l'existence de goîtres.

Nous avons voulu reprendre l'étude du mécanisme d'action du calcium en tant qu'éventuel goitrogène, ayant maintenant à notre disposition la possibilité de doser, par des techniques radio-immunologiques, les hormones thyroïdiennes. Nous avons étudié les hormones thyrö̈diennes dans la glande elle-même, dans le sang et dans deux tissus périphériques chez des rats qui avaient reçu un régime adéquat $(0,47 \%)$ ou un régime excessif $(2 \%)$ en calcium. 


\section{Matériel et méthodes.}

Animaux et régimes. - Des rats mâles Sprague-Dawley (Charles River, France) albinos, au sevrage, ont été mis en cage par groupe de 6 . Ils ont eu libre accès à des biberons d'eau désionisée et ont été nourris avec des régimes ayant deux teneurs différentes en iode, pendant 3 semaines. Le premier régime, considéré comme adéquat en iode $(0,5 \mu \mathrm{g} / \mathrm{g})$ est fourni par UAR (Villemoisson-surOrge, France). II renferme $6 \%$ de caséine ; 78,8 \% de sucres, céréales et lipides ; $10,4 \%$ de protéines végétales ; $7,8 \%$ de protéines animales et $3 \%$ de minéraux $(0,47 \%$ de calcium). Le régime pauvre en iode $(0,04 \mu \mathrm{g} / \mathrm{g})$ est un régime Remington modifié, préparé par I'INRA, (Fabrique de Mélanges alimentaires expérimentaux, 78280, La Minière, France). Ce régime contient $85,5 \%$ de farine de maïs ; $2,32 \%$ de farine de blé avec $70 \%$ de gluten ; $0,23 \%$ de levure de bière ; $4,4 \%$ de lait écrémé non sucré ; $3,48 \%$ de $\mathrm{CaCO}_{3}$ et $1,16 \%$ de $\mathrm{NaCl}$. Des expériences ont été effectuées avec chacun de ces deux régimes enrichis ou non en calcium par addition de $\mathrm{CaCO}_{3}$ (teneurs finales en calcium : 0,47 et $2 \%$ ).

Une autre série d'expériences a été effectuée en utilisant les deux régimes contenant $0,47 \%$ de calcium auxquels a été ajouté $0,1 \%$ de 6 propyl-2thiouracile (PTU). La vitamine $D_{3}(1,5 \mu \mathrm{g} / 0,1 \mathrm{ml})$ a été administrée à tous les animaux par voie orale deux fois par semaine. La vitamine était dissoute dans de I'huile de noix.

Les teneurs en iode total et en calcium ont été mesurées, après minéralisation par les méthodes citées, après chaque livraison.

Procédés expérimentaux. - On administre aux rats par voie intrapéritonéale $24 \mathrm{~h}$ avant le sacrifice de 1 à $2,5 \mu \mathrm{Ci}$ de ${ }^{125}$. Après anesthésie à l'éther, les thyroïdes et les trachées sont prélevées. La fixation de l'iode sur chaque trachée est vérifiée ; elle doit être inférieure à $1 \%$ de celle de la thyroïde. Le sang provient des veines jugulaires et des artères carotides. Les dosages sont effectués sur le sérum après extraction par 2 volumes d'éthanol absolu. Les thyroïdes sont homogénéisées par 1, 2 ou 3 dans $\mathrm{NaCl}$ 0,9\%. Une partie de l'homogénat est hydrolysée en présence de pronase (Etling, 1977).

Les foies et les reins sont prélevés rapidement, mis à $4^{\circ} \mathrm{C}$, pesés et étudiés dans les $24 \mathrm{~h}$, ou congelés à $-20^{\circ} \mathrm{C}$ pour 2 ou 3 jours. Nous avons vérifié qu'une durée de congélation de 1 mois ne modifiait pas les résultats. Nous avons également vérifié que les résultats étaient identiques, que les foies aient été ou non perfusés $30 \mathrm{~min}$ avec $\mathrm{NaCl} 0,9 \%$.

Un rein entier, ou bien $500 \mathrm{mg}$ de foie, sont broyés au moyen d'un Polytron

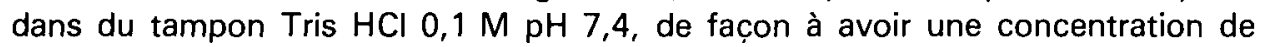
$150 \mathrm{mg}$ de tissu/ml de broyat dans le tampon. Puis les broyats sont extraits par 2 volumes d'éthanol absolu, et centrifugés à $3000 \mathrm{~g}, 10 \mathrm{~min}$ à $6{ }^{\circ} \mathrm{C}$. Les résultats sont exprimés par $\mathrm{g}$ de tissu frais. Les surnageants alcooliques secs des sérums et des tissus sont repris par les tampons utilisés pour effectuer les dosages radioimmunologiques.

Méthodes analytiques. - La concentration du calcium sérique est déterminée au moyen d'un titrateur Marius (Amsterdam, Pays-Bas), et celle du phosphore par la méthode de Fiske et Subbarow adaptée à l'Auto Analyzer Technicon. 
L'iode stable est mesuré par une microméthode (sensibilité 0,5 à $20 \mathrm{ng}$ par $0,1 \mathrm{ml}$ d'échantillon) sur un Auto Analyzer Technicon. L'iode radioactif ${ }^{125}$ est déterminé au moyen d'un compteur à scintillation. La thyroxine $\left(T_{4}\right)$ et la triiodothyronine $\left(T_{3}\right)$ radioactives proviennent d'Amersham (Grande-Bretagne). Les dosages radioimmunologiques de $T_{3}$ sont effectués selon Roux et al. (1975), et ceux de $T_{4}$ selon la méthode préconisée par l'Institut Pasteur (1976); la sensibilité des deux méthodes est de 50 à $500 \mathrm{pg}$ par $0,1 \mathrm{ml}$ d'échantillon. Après extraction des sérums les pourcentages de récupération sont de $96 \%$ pour la $T_{3}$ et de $78 \%$ pour la $\mathrm{T}_{4}$; après extraction des broyats de foie et de reins, les pourcentages récupérés sont respectivement de $83,5 \%$ pour la $T_{3}$ et de $78 \%$ pour la $T_{4}$.

Les iodoaminoacides stables et radioactifs des tissus thyroïdiens hydrolysés sont aussi mesurés après chromatographie sur papier ; les détails de ces techniques ont été décrits antérieurement (Etling, 1977).

Statistiques. - Les moyennes et les erreurs standard $(M \pm S E M)$ sont calculées pour chaque groupe de données lorsqu'il y a au moins 6 valeurs. Les résultats obtenus chez des rats nourris avec un régime renfermant un excès de calcium sont comparés avec ceux de leurs contrôles respectifs en utilisant le test de Student.

\section{Résultats.}

Les effets d'un apport excessif en calcium ( $2 \%)$ dans deux situations (régime adéquat en iode $: 0,5 \mu \mathrm{g} / \mathrm{g}$, et régime pauvre en iode $: 0,04 \mu \mathrm{g} / \mathrm{g}$ ) ont été étudiés sur les paramètres thyroïdiens et périphériques de la fonction thyroïdienne. Ces effets ont été comparés à ceux du PTU, agent goitrogène connu.

A-Action du PTU, lorsque le régime a une teneur adéquate en iode (tabl. 1, 2 premières colonnes). - Le poids de l'animal est inférieur, en présence de PTU, à

TABLEAU 1

Actions du PTU et du calcium ajoutés à un régime renfermant 0,5 $\mu . g$ d'iode par gramme.

\begin{tabular}{|c|c|c|c|c|c|c|c|}
\hline \multirow[b]{2}{*}{$\begin{array}{l}\text { Poids rats }(\mathrm{g}) \ldots \ldots \\
\text { Poids thyroïde }(\mathrm{mg} / \mathbf{1 0 0} \mathrm{g}) \ldots \ldots \ldots \\
\text { Fixation thyroïdienne }(\% \text { dose }) \ldots \\
\text { lode thyroïdien }(\mathrm{ng} / \mathrm{mg}) \ldots \ldots \ldots \ldots \\
\mathrm{T}_{4} \text { thyroïde }(\mathrm{ng} / \mathrm{mg}) \ldots \ldots \ldots \ldots \ldots \\
\mathrm{T}_{3} \text { thyroïde }(\mathrm{ng} / \mathrm{mg}) \ldots \ldots \ldots \ldots \ldots\end{array}$} & $\begin{array}{c}\text { PTU } \\
(0,47 \% \text { Ca) }\end{array}$ & \multicolumn{3}{|c|}{$0,47 \% \mathrm{Ca}$} & \multicolumn{3}{|c|}{$2 \% \mathrm{Ca}$} \\
\hline & $\begin{aligned} 143,9 & \pm 5,3 \\
32,7 & \pm 5,2 \\
3,8 & \pm 1,2 \\
7,2 & \pm 0,9 \\
0,09 & \pm 0,01 \\
0,03 & \pm 0,001\end{aligned}$ & $\begin{array}{r}250,0 \\
7,8 \\
18,9 \\
406,0 \\
66,4 \\
7,6\end{array}$ & $\begin{array}{l} \pm 4,1 \\
\pm 0,4 \\
\pm 0,9 \\
\pm 1,7 \\
\pm 8,8 \\
\pm 0,9\end{array}$ & * & $\begin{array}{r}232,6 \\
8,5 \\
19,5 \\
340,6 \\
67,2 \\
7,7\end{array}$ & $\begin{array}{l} \pm \\
\pm \\
\pm \\
\pm \\
\pm \\
\pm\end{array}$ & $\begin{array}{r}6,0 \\
0,2 \\
1,2 \\
23,6 \\
7,0 \\
0,8\end{array}$ \\
\hline $\begin{array}{l}\text { Ca sérique }(\mathrm{mg} / \mathrm{dl}) \ldots \ldots \ldots \ldots \ldots \\
\text { Radioactivité }(\% \text { dose } / \mathrm{ml}) \ldots \ldots \ldots \ldots \ldots \ldots \ldots \ldots \\
\mathrm{T}_{4} \text { sérique }(\mu \mathrm{g} / \mathrm{dl}) \ldots \ldots \ldots \ldots \ldots \ldots \\
\mathrm{T}_{3} \text { sérique }(\mathrm{ng} / \mathrm{dl}) \ldots \ldots \ldots \ldots\end{array}$ & $\begin{array}{c}10,4 \pm 0,1 \\
0,28 \pm 0,02 \\
<0,02 \pm \\
16,8 \pm 1,0\end{array}$ & $\begin{array}{c}10,4 \\
0,13 \\
3,6 \\
68,5\end{array}$ & $\begin{array}{l} \pm 0,1 \\
\pm 0,01 * \\
\pm 0,3 \\
\pm 2,8\end{array}$ & & $\begin{array}{c}10,6 \\
0,19 \\
4,0 \\
71,5\end{array}$ & $\begin{array}{l} \pm \\
\pm \\
\pm \\
\pm\end{array}$ & $\begin{array}{l}0,12 \\
0,01 \\
0,02 \\
2,0\end{array}$ \\
\hline $\begin{array}{l}\mathrm{T}_{4} \text { hépatique }(\mathrm{ng} / \mathrm{g}) \ldots \ldots \ldots \ldots \ldots \\
\mathrm{T}_{3} \text { hépatique }(\mathrm{ng} / \mathrm{g}) \ldots \ldots \ldots \ldots \ldots \ldots \\
\mathrm{T}_{4} \text { rénale }(\mathrm{ng} / \mathrm{g}) \ldots \ldots \ldots \ldots \ldots \ldots \ldots \\
\mathrm{T}_{3} \text { rénale }(\mathrm{ng} / \mathrm{g}) \ldots \ldots \ldots \ldots \ldots \ldots\end{array}$ & $\begin{array}{l}0,44 \pm 0,03 \\
0,49 \pm 0,05 \\
0,46 \pm 0,04 \\
0,29 \pm 0,02\end{array}$ & $\begin{array}{c}24,9 \\
2,95 \\
12,1 \\
4,33\end{array}$ & $\begin{array}{l} \pm 1,8 \\
\pm 0,16 \\
\pm 0,7 \\
\pm 0,12\end{array}$ & ** & $\begin{array}{c}32,8 \\
2,77 \\
14,3 \\
4,8\end{array}$ & $\begin{array}{l} \pm \\
\pm \\
\pm \\
\pm\end{array}$ & $\begin{array}{l}2,4 \\
0,11 \\
0,6 \\
0,2\end{array}$ \\
\hline
\end{tabular}

Moyennes \pm SEM. Comparaisons entre l'effet de 0,47 et $2 \%$ de $\mathrm{Ca}:{ }^{*} \mathrm{P}<0,01 ;{ }^{*} \mathrm{P}<0,02$; *** $\mathbf{P}<0,001$. 
celui des animaux témoins; le poids de la thyroïde est plus élevé lorsqu'il est exprimé par rapport au poids de l'animal. Les teneurs en hormones du tissu thyroïdien et leurs concentrations sériques sont effondrées. Les hormones contenues dans les tissus hépatiques et rénaux sont aussi en concentration inférieure à celle des tissus d'animaux témoins.

Les mêmes effets sont observés lorsque le PTU est administré à des rats nourris avec un régime pauvre en iode (tabl. 2, 2 premières colonnes).

$B$-Action du calcium, lorsque le régime a une teneur adéquate en iode $(0,5 \mu \mathrm{g} / \mathrm{g})$. - Les rats qui consomment un excès de calcium ont un poids significativement inférieur $(p<0,01)$ à ceux qui reçoivent un régime normal en calcium (tabl. 1). L'excès de calcium ne modifie ni le poids des thyroïdes (rapporté au poids du rat), ni la fixation thyroïdienne de l'iode radioactif, ni les teneurs en hormones du tissu thyroïdien. Par contre, la teneur en iode du tissu thyroïdien est significativement plus basse $(p<0,01)$ que chez les animaux recevant un régime normal en calcium. La séparation des iodoaminoacides thyroïdiens par chromatographie donne des résultats qui confirment ceux obtenus par dosage radioimmunologique. On constate que la diiodotyrosine est 2 fois plus abondante que la monoiodotyrosine et que la $\mathrm{T}_{4}$ représente au maximum $10 \%$ de l'iode total, marqué ou non. L'excès de calcium du régime ne modifie pas ces résultats.

A la périphérie, l'excès de calcium n'a pas modifié la calcémie. La radioactivité sérique est significativement plus élevée ( $p<0,001$ ) que chez les rats ayant reçu un régime adéquat en calcium. Les concentrations hormonales ne sont pas modifiées. Les teneurs en $\mathrm{T}_{4}$ des tissus hépatiques et rénaux sont significativement plus élevées lorsqu'il y a un excès de calcium (respectivement $p<0,01$ et $p<0,02)$; les teneurs en $T_{3}$ ne sont pas modifiées. Le poids des foies par rapport au poids total de l'animal est de $0,049 \pm 0,008$, le foie fixant $0,77 \pm 0,01 \%$ de la radioactivité administrée si le régime est normal en calcium ; ce rapport est de $0,044 \pm 0,008$, le foie fixant $1,04 \pm 0,09 \%$ de l'activité lorsqu'il y a un excès. de calcium.

C-Action du calcium, lorsque le régime a une teneur faible en iode $(0,04 \mu \mathrm{g} / \mathrm{g})$. - Les animaux nourris 3 semaines avec ce régime enrichi en calcium ont un poids significativement inférieur $(p<0,01)$ à ceux ayant reçu le même régime sans addition de $\mathrm{CaCO}_{3}$ (tabl. 2). L'excès de calcium ne modifie pas le poids des thyroïdes, mais augmente la fixation de l'iode radioactif ( $p<0,05$ ). L'excès de calcium absorbé par les animaux ne modifie ni la teneur en iode du tissu thyroïdien, ni sa teneur en $T_{4}$ et en $T_{3}$. Du fait de la déficience en iode du régime, les pourcentages de l'iode sous forme de monoiodotyrosine (mesurés après chromatographie) sont du même ordre de grandeur que ceux de l'iode de la diiodotyrosine; les pourcentages d'iode hormonal, radioactif et stable, sont, par cette méthode environ $24 \%$ de l'iode total. La teneur en calcium du régime ne modifie pas ces différentes valeurs.

Lorsque le régime est pauvre en iode, l'excès de calcium provoque une augmentation significative $(p<0,001)$ de la calcémie. La surcharge en calcium ne provoque pas de modification de la radioactivité sérique ni de la concentration en $\mathrm{T}_{4}$. Par contre la concentration en $\mathrm{T}_{3}$, élevée à cause de la déficience en iode du régime, lorsque le calcium a une teneur normale, est très significative- 
TABLEAU 2

Actions du PTU et du calcium ajoutés à un régime renfermant $0,04 \mu g$ d'iode par gramme.

\begin{tabular}{|c|c|c|c|c|c|}
\hline \multirow[b]{2}{*}{$\begin{array}{l}\text { Poids rats }(\mathrm{g}) \ldots \ldots \ldots \ldots \\
\text { Poids thyroïde }(\mathrm{mg} / 100 \mathrm{~g}) \ldots \ldots \ldots \\
\text { Fixation thyroïdienne }(\% \text { dose }) \ldots \ldots \\
\text { lode thyroïdien }(\mathrm{ng} / \mathrm{mg}) \ldots \ldots \ldots \ldots \\
\mathrm{T}_{4} \text { thyroïde }(\mathrm{ng} / \mathrm{mg}) \\
\mathrm{T}_{3} \text { thyroïde }(\mathrm{ng} / \mathrm{mg}) \ldots \ldots \ldots \ldots \ldots \ldots\end{array}$} & $\begin{array}{c}\text { PTU } \\
(0,47 \% \mathrm{Ca})\end{array}$ & \multicolumn{2}{|l|}{$0,47 \% \mathrm{Ca}$} & \multicolumn{2}{|c|}{$2 \% \mathrm{Ca}$} \\
\hline & $\begin{aligned} 105,5 & \pm 4,2 \\
58,4 & \pm 5,8 \\
3,6 & \pm 0,4 \\
0,9 & \pm 0,1 \\
0,04 & \pm 0,006 \\
0,02 & \pm 0,003\end{aligned}$ & 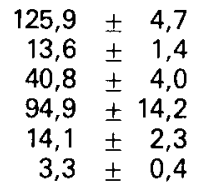 & $\begin{array}{l}* \\
* *\end{array}$ & $\begin{array}{r}103,1 \\
14,8 \\
52,1 \\
101,0 \\
19,4 \\
3,6\end{array}$ & $\begin{aligned} \pm & 4,8 \\
\pm & 0,7 \\
\pm & 2,6 \\
\pm & 12,9 \\
\pm & 3,4 \\
\pm & 0,3\end{aligned}$ \\
\hline $\begin{array}{l}\text { Ca sérique }\{\mathrm{mg} / \mathrm{dl}\} \ldots \ldots \ldots \ldots \\
\text { Radioactivité }(\% \text { dose } / \mathrm{ml}\} \ldots \ldots \ldots \\
\mathrm{T}_{4} \text { sérique }(\mu \mathrm{g} / \mathrm{dl}) \ldots \ldots \ldots \ldots \ldots \ldots \\
\mathrm{T}_{3} \text { sérique }(\mathrm{ng} / \mathrm{dl}) \ldots \ldots \ldots \ldots \ldots\end{array}$ & $\begin{aligned} & 11,0 \\
< & 0,36 \pm 0,01 \\
< & 0,02 \\
< & 15\end{aligned}$ & $\begin{array}{c}11,7 \pm 0,1 \\
1,02 \pm 0,14 \\
3,7 \pm 0,2 \\
205,1 \pm 13,9\end{array}$ & $* * *$ & $\begin{array}{c}12,6 \\
1,11 \\
3,7 \\
46,3\end{array}$ & $\begin{array}{ll} \pm & 0,2 \\
\pm & 0,14 \\
\pm & 0,3 \\
\pm & 11.9\end{array}$ \\
\hline $\begin{array}{l}\mathrm{T}_{4} \text { hépatique }(\mathrm{ng} / \mathrm{g}) \\
\mathrm{T}_{3} \text { hépatique }(\mathrm{ng} / \mathrm{g}) \ldots \ldots \ldots \ldots \ldots \ldots \ldots \\
\mathrm{T}_{4} \text { rénale }(\mathrm{ng} / \mathrm{g}) \ldots \ldots \ldots \ldots \ldots \ldots \ldots \\
\mathrm{T}_{3} \text { rénale }(\mathrm{ng} / \mathrm{g}) \ldots \ldots \ldots \ldots \ldots \ldots\end{array}$ & $\begin{array}{l}1,6 \pm 0,1 \\
0,2=0 \\
0,9 \pm 0,05 \\
0,2\end{array}$ & $\begin{array}{r}13,3 \pm 0,1 \\
3,3 \pm 0,1 \\
9,8 \pm 0,8 \\
4,5 \pm 0,3\end{array}$ & * & $\begin{array}{r}15,1 \\
2,6 \\
9,2 \\
3,1\end{array}$ & $\begin{array}{l} \pm 1,1 \\
\pm \quad 0,1 \\
\pm \quad 0,8 \\
\pm \quad 0,3\end{array}$ \\
\hline
\end{tabular}

Moyennes \pm SEM. Comparaisons entre l'effet de 0,47 et $2 \%$ de $C a:{ }^{*} P<0,01 ;{ }^{*} P<0,05$; ***P<0,001.

ment abaissée ( $p<0,001)$ lorsque les rats sont nourris avec un régime riche en calcium. Les teneurs en $T_{4}$ des tissus hépatiques et rénaux ne sont pas modifiées par la quantité de calcium du régime. Les teneurs en $T_{3}$ des tissus hépatiques et rénaux sont significativement abaissées $(p<0,01)$ par la surcharge en calcium du régime. Les poids des foies, par rapport aux poids des animaux, ne sont pas différents avec ou sans excès de calcium $10,039 \pm 0,07$ avec calcium normal et $0,033 \pm 0,009$ avec $2 \% \mathrm{Ca}$ ), ainsi que la fixation de ${ }^{125}$ par les foies $(2,8 \pm 0,4 \%$ de l'iode total avec calcium normal et $2,7 \pm 0,2 \%$ avec $2 \% \mathrm{Ca})$.

\section{Discussion.}

L'hypothèse, émise en 1831 par Boussingault, selon laquelle le calcium pourrait agir comme un goitrogène n'a jamais été vérifiée. II semble que les derniers travaux sur ce sujet datent de 1954 (Taylor). La sensibilité des techniques radioimmunologiques, et nos connaissances actuelles sur les effets des hormones thyroïdiennes au niveau cellulaire ont rendu possible une nouvelle approche de ce problème.

Nous avons d'abord examiné l'action du propyithiouracile (PTU), antithyroïdien actif chez l'animal (Mayberry et Astwood, 1960), chez des rats nourris avec deux régimes de teneurs différentes en iode. Si la teneur en iode du régime convient à un métabolisme normal $(0,5 \mu \mathrm{g} / \mathrm{g})$, ou si le régime est pauvre en iode $(0,04 \mu \mathrm{g} / \mathrm{g})$ (Studer et Greer, 1968), la croissance pondérale du rat est lente en présence de PTU ; la glande thyroïde grossit et acquiert rapidement l'aspect histologique du goître. Le tissu thyroïdien contient peu d'iode stable et fixe peu l'iode radioactif ; la synthèse des hormones thyroïdiennes est presque nulle, et les concentrations hormonales sériques sont trop faibles pour être mesurées. Les teneurs 
en thyroxine et en triiodothyronine des tissus hépatiques et rénaux sont à peine détectables. Ces effets sont caractéristiques des goitrogènes classiques.

Un excès de calcium ne modifie ni le poids des glandes thyroïdes, ni la teneur en thyroxine et en triiodothyronine du tissu thyroidien quelle que soit la teneur en iode du régime. L'excès de calcium provoque une modification de la teneur en iode du tissu thyroïdien et de la fixation de l'iode marqué, mais ces effets dépendent de la teneur en iode du régime.

A la périphérie, lorsque le régime a une teneur satisfaisante en iode, un excès de calcium provoque une augmentation de la radioactivité sérique et de la teneur en thyroxine des tissus hépatiques et rénaux. Les teneurs en hormones que nous avons obtenues dans ces tissus sont très voisines de celles de Van Doorn, Roelfsema et Van der Heide (1985), qui utilisent une méthode de dosage par équilibre isotopique. L'excès de calcium ne provoque aucun changement de la teneur en triiodothyronine des tissus hépatiques et rénaux; celles-ci sont un peu inférieures à celles de Van Doorn, Roelfsema et Van der Heide (1985) mais proches de celles de Obregon, Morreale de Escobar et Escobar del Rey (1978). Ces auteurs trouvent, comme nous, une teneur en triiodothyronine plus élevée dans le tissu rénal que dans le tissu hépatique. Ces résultats diffèrent de ceux de Nejad et al. (1975) qui trouvent des teneurs en triiodothyronine plus élevées que les nôtres, surtout dans le tissu hépatique. Tous les résultats montrent donc que lorsque le régime a une teneur adéquate en iode, le calcium n'induit aucun des critères de la goitrogenèse.

Lorsque le régime est pauvre en iode, l'excès de calcium a un effet très particulier : il annule partiellement l'augmentation de la concentration sérique de triiodothyronine, due à la déficience en iode (Studer et Greer, 1968). Cette diminution, de l'ordre de $29 \%$, est un phénomène rapide. En effet au bout d'une semaine, les animaux nourris avec un régime ayant une teneur normale en calcium, ont une concentration sérique de triiodothyronine de 157,3 $\pm 5 \mathrm{ng}$ par $\mathrm{dl}$, et en présence d'un excès de calcium la concentration de triiodothyronine n'est que de $124,1 \pm 13 \mathrm{ng} / \mathrm{dl}$. Cette diminution est probablement provoquée par une inhibition de la conversion de la thyroxine en triiodothyronine. La diminution de la quantité de triiodothyronine est une modification importante puisque c'est cette hormone qui est active au niveau cellulaire. La teneur en thyroxine des tissus hépatiques et rénaux n'est pas modifiée par l'excès de calcium, mais avec un excès de calcium les quantités de triiodothyronine renfermées par les tissus hépatiques sont diminuées de $21 \%$, celles des tissus rénaux de $33 \%$. L'inhibition de la conversion de la thyroxine dans le tissu rénal est plus élevée que celle notée dans le sérum. Ce fait expérimental peut être rapproché de résultats récents (Van Doorn, Roelfsema et Van der Heide, 1985) montrant que la présence de triiodothyronine dans le tissu rénal proviendrait surtout du plasma, et que celle de triiodothyronine dans le tissu hépatique (moins abondante) proviendrait de la conversion locale de la thyroxine : ceci pourrait expliquer la cause de la plus faible inhibition de conversion dans le tissu hépatique.

Le foie et le rein sont des organes cibles qui réagissent aux hormones thyroïdiennes (Barker, 1955). Nous avons recherché si, le calcium pouvait inhiber aussi la conversion de la thyroxine dans les organes qui ne sont pas des organes cibles 
classiques. La rate et le muscle, qui appartiennent à cette catégorie, contiennent, lorsque le régime est pauvre en iode, moins de $1 \mathrm{ng}$ de triiodothyronine par gramme ce qui ne permet pas de mettre en évidence une éventuelle action du calcium.

Au total, l'excès d'apport nutritionnel en calcium modifie chez le rat la fonction thyroïdienne mais ne paraît pas avoir d'action goitrogène. L'effet du calcium sur la fonction thyroïdienne pourrait avoir comme intermédiaire une inhibition de la conversion de la thyroxine en triiodothyronine dans le sang et les tissus périphériques. A l'inverse, la thyroïde pourrait elle-même moduler la capacité du rat à maintenir sa calcémie dans les limites normales. En effet, la calcémie des rats en régime adéquat en calcium et la réponse de cette calcémie à une surcharge chronique en calcium, étaient plus élevées chez les rats déficients en iode que chez les rats ayant reçu un apport adéquat en iode. Une étude statistique de l'effet de l'apport nutritionnel en iode sur l'homéostasie calcique n'a pas été possible du fait que le contenu en iode n'était pas la seule différence entre les deux régimes ; un tel effet pourrait être étudié.

Reçu en janvier 1986.

Accepté en mars 1986.

Remerciements. - A Michèle Poitou pour son aide efficace apportée lors de la préparation du manuscrit.

\section{Références}

BARKER S. B., 1955. Effect of thyroid status on succinate oxydation by various tissues of the rat. Endocrinology, 57, 414-418.

BOUSSINGAULT J. B., 1831. Recherches sur la cause qui produit le goître dans les Cordillères de la Nouvelle-Grenade. Ann. Chim. Phys., 48, 41-69.

ETLING N., 1977. Concentration of thyroglobulin, iodine content of thyroglobulin and of iodoaminoacids in human neonates thyroid glands. Acta paediatr. scand., 66, 97-102.

INSTITUT PASTEUR PRODUCTION, 1976. Unité de radio-immunologie ana/ytique (Fiche 79.680).

LANGER P., GREER M. A., 1977. Chemical classification of antithyroid substances. In Antithyroid substances and naturally occurring goitrogens. Karger S. Ed., Basel.

MAYBERRY W. E., ASTWOOD E. B., 1960. The effect of propylthiouracil on the intrathyroid metabolism of iodine in rats. J. biol. Chem., 235, 2977-2980.

NEJAD I., BOLLINGER J., MITNICK M. A., SULLIVAN P., REICHLIN S., 1975. Measurement of plasma and tissue triiodothyronine concentration in the rat by radioimmunoassay. Endocrinology, 96, 773-780.

OBREGON M. J., MORREALE DE ESCOBAR G., ESCOBAR DEL REY F., 1978. Concentrations of triiodo-1-thyroxine in the plasma and tissues of normal rats as determined by radioimmunoassay : comparison with results obtained by an isotopic equilibrium technique. Endocrinology, 103, 2145-2153.

ROUX D., PEYRIN J. O., CHAMBOSSE A., BORNET H., 1975. Dosage radio immunologique sans extraction de la l-triiodothyronine dans le sérum humain. C.R. Soc. Biol., 169, 354-358.

SHARPLESS G. R., SABOL M., ANTONY E. K., ARGETSINGER H. L., 1943. Goitrogenic action of calcium and vitamin D. J. Nutr., 25, 119-126.

STUDER H., GREER M. A., 1968. The regulation of thyroid function in iodine deficiency. Hans Huber Pub, Berne.

TAYLOR S., 1954. Calcium as a goitrogen. J. clin. Endocrinol. Metab., 14, 1412-1422.

VAN DOORN J., ROELFSEMA F., VAN DER HEIDE D., 1985. Concentrations of thyroxine and 353' triiodothyronine at 34 different sites in euthyroid rats as determined by an isotopic equilibrium technique. Endocrinology, 117, 1201-1208. 